\title{
Multi-Material Binder Jet Printing of Functional Ni-Mn-Ga Alloys
}

\author{
Erica Stevens, Katerina Kimes, Jeffrey Martin and Markus Chmielus
}

University of Pittsburgh, Pittsburgh, Pennsylvania, United States

Additive manufacturing has the well-known benefits of reduced waste material and geometrical freedom in parts. Powder bed binder jet 3D printing is a type of additive manufacturing that is versatile, is not energy based, operates in air at room temperature, and allows for controlled densification during postprocessing. In BJ-3DP, a layer of powder is spread flat by a roller on a build bed. A printhead selectively deposits binder onto the powder in a pre-determined path for that layer. The build bed moves down by one layer thickness, another layer of powder is spread, the binder is deposited, and the process repeats until the part is complete. After printing, the entire build bed of powder is heated in an oven to fully cure the binder, after which the parts can be handled but are still approximately 50-60\% dense [1,2]. For metals, a common post-processing option is pressureless sintering, using only high temperatures to allow the metal powders to densify into a solid part that is $70-100 \%$ dense $[1,3]$.

$\mathrm{Ni} 2 \mathrm{MnGa}$ off-stoichiometric Heusler alloys have been heavily researched as functional magnetic materials $[4,5]$. Their solid-state phase transformation between low temperature martensite and high temperature austenite gives rise to the existence of, for example, a magnetic shape memory effect or a magnetocaloric effect. The magnetocaloric effect in particular (when a material changes temperature with adiabatic magnetization) is most effective when materials are layered in order of their Curie temperatures [6]. BJ3DP may be beneficial for producing magnetocaloric compositions of Ni-Mn-Ga alloys due to the lack of melting during printing and the ability to design complex geometries for heat exchangers, but the ability to layer different compositions to create a gradient of Curie temperatures is currently missing.

This study used a self-designed and self-built manual binder jet 3D printer capable of accommodating multiple materials into a single build. Single-composition and stacked layer samples of Ni49.7Mn30.0Ga20.3 and $\mathrm{Ni49.6} \mathrm{Mn}_{19.2} \mathrm{Ga}_{6.3} \mathrm{Cu}_{24.9}$ (at.\%) were produced and analyzed for thermal properties and compositional variations.

Multi-layered printing was tested by using the Ni-Mn-Ga and a Cu-doped Ni-Mn-Ga composition in each of the powder feeders in the manual binder jet 3D printer. 16 total $250 \mu \mathrm{m}$ thick layers were printed, in 4layer increments of each material. Printing of these same pure powders and sintering at $1080{ }^{\circ} \mathrm{C}$ showed very distinct martensitic transformation temperature ranges (Fig. 1), from 5-22 ${ }^{\circ} \mathrm{C}$ for the $\mathrm{Ni} 49.6 \mathrm{Mn}_{19.2} \mathrm{Ga}_{6.3} \mathrm{Cu}_{24.9}$ and $60-102{ }^{\circ} \mathrm{C}$ for the $\mathrm{Ni} 49.7 \mathrm{Mn}_{30.0} \mathrm{Ga}_{20.3}$. The printed layered structure retained two distinct peaks, at $45-58{ }^{\circ} \mathrm{C}$ and $58-82{ }^{\circ} \mathrm{C}$, though these phase transformation temperatures were significantly shifted from the pure samples due to a high level of sensitivity to composition in these alloys.

Layers in the multi-layered printing were also visually distinct as in the SEM micrograph in Fig. 2, mainly due to differences in densification since the $\mathrm{Cu}$ addition lowers the melting point. There was some diffusion of elements confirmed by EDS elemental analysis, the reason for the phase transformation temperature shift in DSC data. However, there were points with 0 at.\% $\mathrm{Cu}$ in the center of the Ni-Mn-Ga layer, indicating that powder mixing did not occur during the printing process. Continued work includes binder deposition optimization and testing with different particle sizes and shapes [7]. 


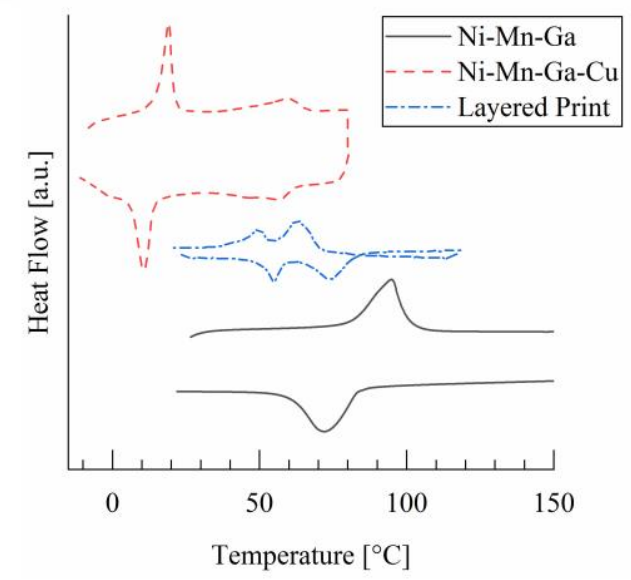

Figure 1. Thermal transformation data for $\mathrm{Ni}-\mathrm{Mn}-\mathrm{Ga}, \mathrm{Cu}$-doped $\mathrm{Ni}-\mathrm{Mn}-\mathrm{Ga}$, and a layered print comprised of both materials.

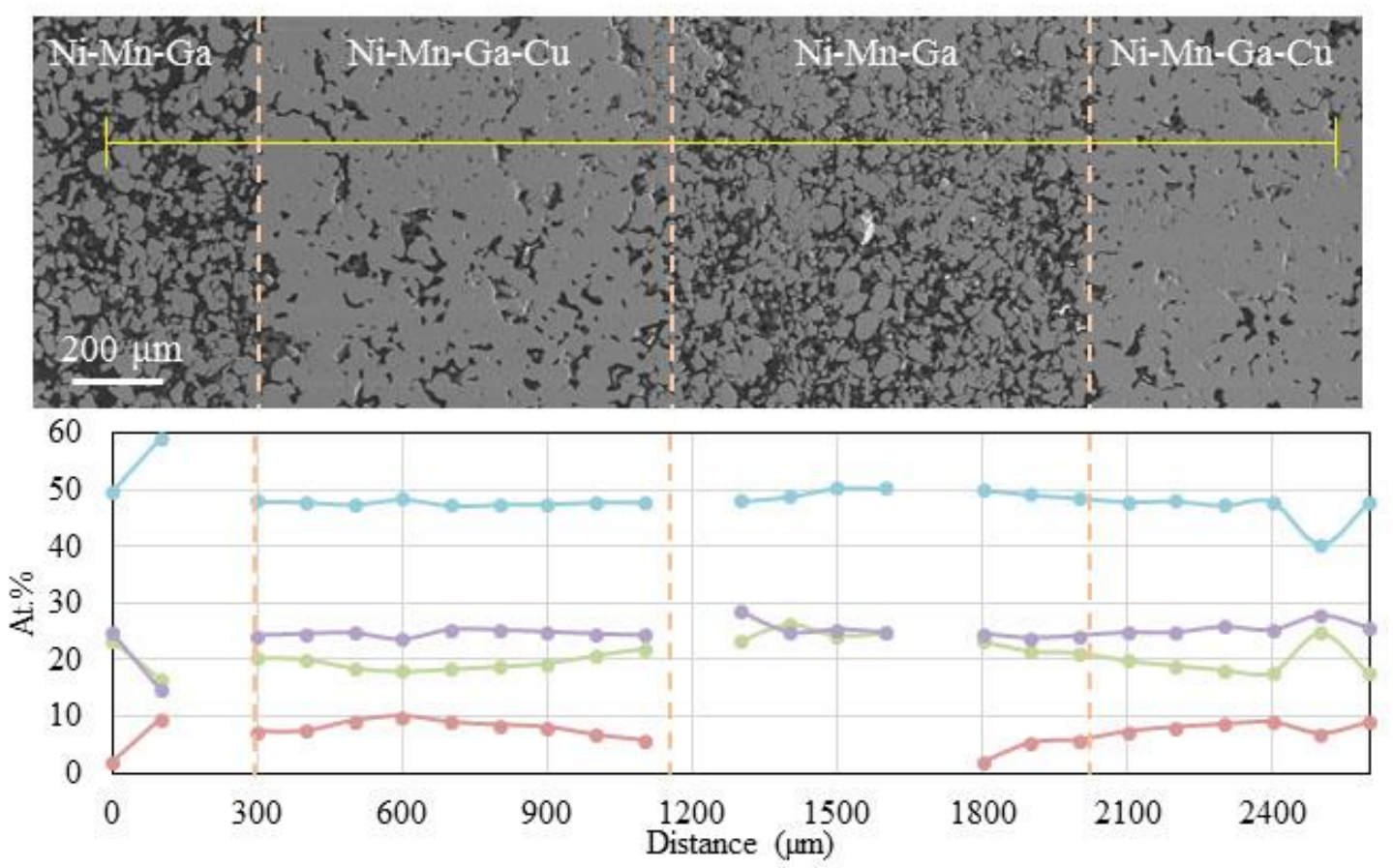

Figure 2. Composition analysis along a line through the layered sample cross-section. Data was collected at each indicated point.

\section{References}

[1] Mostafaei, Amir, Erica L. Stevens, Eamonn T. Hughes, Shannon D. Biery, Colleen Hilla, and Markus Chmielus. "Powder Bed Binder Jet Printed Alloy 625: Densification, Microstructure and Mechanical Properties." Materials \& Design $108 \quad$ (October 2016): 126-35. https://doi.org/10.1016/j.matdes.2016.06.067.

[2] Mostafaei, Amir, Katerina A. Kimes, Erica L. Stevens, Jakub Toman, Yuval L. Krimer, Kari Ullakko, and Markus Chmielus. "Microstructural Evolution and Magnetic Properties of Binder Jet Additive Manufactured Ni-Mn-Ga Magnetic Shape Memory Alloy Foam.” Acta Materialia 131 (June 2017): 48290. https://doi.org/10.1016/j.actamat.2017.04.010. 
[3] Stevens, Erica, Samantha Schloder, Eric Bono, David Schmidt, and Markus Chmielus. "Density Variation in Binder Jetting 3D-Printed and Sintered Ti-6Al-4V." Additive Manufacturing 22 (August 2018): 746-52. https://doi.org/10.1016/j.addma.2018.06.017.

[4] Franco, V., J. S. Blázquez, J. J. Ipus, J. Y. Law, L. M. Moreno-Ramírez, and A. Conde. "Magnetocaloric Effect: From Materials Research to Refrigeration Devices." Progress in Materials Science 93 (2018): 112-232. https://doi.org/10.1016/j.pmatsci.2017.10.005.

[5] Mostafaei, Amir, Pierangeli Rodriguez De Vecchis, Erica L. Stevens, and Markus Chmielus. "Sintering Regimes and Resulting Microstructure and Properties of Binder Jet 3D Printed Ni-Mn-Ga Magnetic Shape Memory Alloys." Acta Materialia 154 (August 2018): 355-64. https://doi.org/10.1016/j.actamat.2018.05.047.

[6] Zimm, Carl, Andre Boeder, Bryant Mueller, Kyle Rule, and Steven L. Russek. "The Evolution of Magnetocaloric Heat-Pump Devices." MRS Bulletin 43, no. 4 (2018): 274-79. https://doi.org/10.1557/mrs.2018.71.

[7] This work was performed, in part, at the Nanoscale Fabrication and Characterization Facility, a laboratory of the Gertrude E. and John M. Petersen Institute of NanoScience and Engineering and at the Materials Micro-Characterization Laboratory containing the Fishione Center of Excellence for EMsample Preparation. This project was supported in part by a fellowship award through the National Defense Science and Engineering Graduate (NDSEG) Fellowship Program, sponsored by the Air Force Research Laboratory (AFRL), the Office of Naval Research (ONR) and the Army Research Office (ARO). Funding for JM was provided by the University of Pittsburgh Swanson School of Engineering. Special thanks to Grant Merkel for design contributions, and to Andy Holmes and the Swanson Center for Product Innovation for the assistance and tooling necessary to complete a functioning device. 